\title{
La formación del sistema hegeliano. Una sistematización de los escritos de juventud
}

\author{
Edgar F. Rodríguez Aguilar \\ Academia de Ciencia Política y Administración Urbana \\ Universidad Autónoma de la Ciudad de México \\ hadesera@yahoo.com
}

\begin{abstract}
La filosofía es la ciencia objetiva de la verdad, la ciencia de su necesidad, de su conocer reducido a conceptos, y no un simple opinar o devanar de opiniones.
\end{abstract}

Hegel

En una conocida carta fechada el 2 de noviembre de 1800, Hegel escribe a Schelling: «Mi formación científica comenzó por necesidades humanas de carácter secundario; así tuve que ir siendo empujado hacia la Ciencia, y el ideal juvenil tuvo que ir tomando la forma de la reflexión, convirtiéndose en sistema» ${ }^{1}$. Esta carta es reveladora del paso de los escritos hegelianos previos a 1800, a las redacciones del autor que acompañan el nacimiento del siglo XIX.

A primera vista, podríamos pensar que la obra de juventud de Hegel está radicalmente escindida de los trabajos comenzados a partir de su llegada a Jena, momento en el que emprendería la empresa de la que le habla a Schelling en la carta citada, y que comprende la obra de madurez. Basta echar un vistazo a algunas páginas de los Escritos de juventud y compararlas con cualquier párrafo de la Fenomenología del espíritu, publicada en 1807, para constatar la diferencia en los estilos de la escritura, la rigidez en la forma de tratar los temas presentados y la linealidad - en el sentido del hilo conductor - que guía su interpretación. La prosa relajada con la que están construidos los Escritos de juventud le permite a Hegel ir

${ }^{1}$ G. W. F. HeGeL, «Carta a Schelling», en: Escritos de juventud, Madrid: FCE 1978, p. 433. 
y venir de un tema a otro, interpretar libremente pasajes del $A n$ tiguo y Nuevo Testamento y criticar dura - y en algunas ocasiones viseralmente - las acciones de los distintos personajes que aparecen en ellos. Pero en la Fenomenología del espíritu aparece aquella oscuridad lingüística que tanto preocupó a Goethe en función del conocimiento que la gente tendría de la filosofía alemana, los temas tratados son expuestos de manera sistemática y hay un cierto cuidado en las interpretaciones realizadas. Esta transformación en la obra hegeliana ciertamente responde a maneras distintas de comprender y emprender el quehacer filosófico, pero no a distintos objetos que se traten en él.

Esta es la propuesta interpretativa del presente texto: si bien, como menciona Lukács, el tema central de los Escritos de juventud es la positividad del cristianismo y el de la Fenomenología del espíritu es el de la reconciliación de los opuestos ${ }^{2}$, la diferencia no estriba en el objeto del que trata el trabajo filosófico, sino en la manera de su tratamiento. Nos parece que tanto en los Escritos de juventud como en el sistema propiamente dicho, el cual -más por casualidades y azares inmediatos que por un proyecto intelectual o académico así definido - comienza con la Fenomenología del espíritu, el objeto a tratar es el mismo: el absoluto, mas las formas de su tratamiento variarán significativamente y darán por resultado aquello que a primera vista aparece como dos proyectos filosóficos radicalmente distintos, o incluso uno toda vez que los escritos juveniles ni siquiera sean tomados como un proyecto filosófico.

La manera en que Hegel aborda la presencia del absoluto en el mundo a partir de 1800 es describiendo, desde la forma más acabada alcanzada por éste - a saber el concepto filosófico-, el camino recorrido en su propio proceso de formación, hasta ser saber de sí mismo. Desde comienzos del siglo XIX y en adelante, Hegel adopta aquella forma de la filosofía descrita años más tarde en el Prefacio de Los principios de la filosofía del derecho con las siguientes palabras:

[...] la filosofía llega siempre demasiado tarde. En cuanto pensamiento del mundo, aparece en el tiempo sólo después de que la realidad ha

2 Cf. Georg LuKács, El joven Hegel y los problemas de la sociedad capitalista, Barcelona: Grijalbo 1972. 
consumado su proceso de formación y se halla ya lista y terminada. Lo que enseña el concepto lo muestra con la misma necesidad la historia: sólo en la madurez de la realidad aparece lo ideal frente a lo real y lo erige a este mismo mundo, aprehendido en su sustancia, en la figura de un reino intelectual. Cuando la filosofía pinta con sus tonos grises, ya ha envejecido una figura de la vida que sus penumbras no pueden rejuvenecer, sino sólo conocer; el búho de Minerva sólo alza su vuelo en el ocaso ${ }^{3}$.

Bajo esta idea de filosofía no hay lugar para imponerle a la realidad, ni siquiera demandarle, algún tipo de libertad o racionalidad externa a ella misma; no hay lugar para enfrentarla a nada que le sea externo y extraño, sino que sólo cabe realizar la descripción de las contradicciones que le son inherentes: sus formas íntimas de escisión, mediación y superación. Así es como Hegel opera, por lo menos desde la Fenomenología del espíritu hasta sus últimas lecciones dictadas en Berlín, para aprehender filosóficamente la presencia del absoluto en los ámbitos lógico, del saber, de la historia factual, de la historia de la filosofía, de las formas religiosas y las figuras artísticas, y la constitución del Estado.

A lo largo de su obra considerada de madurez, Hegel se opone a toda perspectiva que no sea coherente con la vigencia de la realidad, con aquellos proyectos tanto filosóficos como políticos que conciben formas de libertad y racionalidad que no le corresponden a la realidad, e incluso intentan imponérselas aunque sea de manera forzada. De aquí la separación de Hegel tanto de Kant como de Robespierre $^{4}$.

Sin embargo, a esta manera fenomenológica y crepuscular de buscar la aprehensión del absoluto, Hegel no llega de manera espontánea, sino sólo gracias al camino emprendido durante su época de juventud. En ella, la manera en que Hegel realiza el quehacer filosófico es radicalmente opuesta a como la lleva a cabo a partir de 1800. Pero como hemos mencionado, es la forma de llevar a cabo dicho camino la que se presenta como distinta, e incluso opuesta, a

${ }^{3}$ G. W. F. Hegel, Principios de la filosofía del derecho, Barcelona: Edhasa 1999, p. 63.

${ }^{4}$ Cf. Jürgen Habermas, Teoría y praxis, Madrid: Ediciones Altaya 1999, caps. 3 y 4. 
su comprensión de la filosofía, mas no el problema a tratar en ella.

Ahora bien, esta perspectiva aún no fenomenológica desde la que se intenta abordar la aprehensión del absoluto en los años de juventud - marcada claramente por el sistema crítico kantiano y la Revolución francesa - pasa por distintas etapas. En la primera de ellas, que va aproximadamente de 1793 a 1796, Hegel enfrenta la estructura dogmática y heterónoma del cristianismo con el entusiasmo provocado por el Sapere aude! kantiano, y realiza una crítica, tomando como parámetro el concepto de «autonomía de la razón», tanto al judaísmo como a la manera en que el desarrollo del cristianismo tergiversó las enseñanzas de Jesús. Opone la ahistoricidad y, como lo llamará más tarde, el carácter abstracto de la autonomía kantiana de la voluntad a las condiciones sociales de la época a través de su lectura de la vida de Jesús. Principalmente en Historia de Jesús, de 1794, y «La positividad de la religión cristiana», redactada entre 1794 y 1795, Hegel hace una interpretación radicalmente kantiana de las enseñanzas de Jesús en la que éste aparece como encarnación del imperativo categórico; como un revolucionario que, solo en el mundo, enfrenta la heteronomía de su época al carácter autónomo de la voluntad racional. En Los principios de la filosofía del derecho Hegel, habiendo asumido ya la idea crepuscular y fenomenológica de la filosofía, advierte que «todo hombre no puede más que ser un hijo de su tiempo $»^{5}$. Sin embargo, en los escritos tempranos, Jesús aparece en contracorriente con su tiempo. «La religión [que Jesús] llevó en su corazón - menciona Hegel- estaba libre del espíritu de su pueblo» ${ }^{6}$. A pesar de que la preocupación hegeliana desde estos momentos es la manera en que puede aprehenderse y conocerse el absoluto, y éste, como sabemos, una vez construido el sistema se despliega principalmente en la historia; a pesar de esto, en estos primeros escritos la dimensión histórica no aparece aún. En las obras referidas, el Mesías más que como un hijo de Dios y un hombre de fe aparece como un educador del pueblo que insta a los hombres a no dejarse llevar más que por su propio

${ }^{5}$ Hegel, Principios de la..., p. 61.

${ }^{6}$ G. W. F. Hegel, «La positividad de la religión cristiana [Nuevo comienzo]», en: Escritos de juventud, p. 431. 
entendimiento, sin la guía de otro. Su enseñanza, según Hegel, no consistió en adoctrinar gente presentándole contenidos específicos de formas de vida que debieran seguir al pie de la letra, sino en mostrarles que sólo accediendo al propio llamado interior de su razón podrían ser hijos de Dios. De esta oposición del Mesías al carácter muerto de las letras de los libros sagrados y las leyes de los judíos que, al ser impuestas se muestran como mandatos externos que, por tanto, se asumen de manera heterónoma, se sigue que su único destino lógico haya sido la muerte en la cruz. Ésta para Hegel tiene dos interpretaciones: la representación de la no separación entre los mundos divino y humano, y la muestra del fracaso de la empresa del Mesías tras la victoria de las fuerzas de la historia sobre las obras humanas particulares. Esta segunda interpretación le permite a Hegel reflexionar acerca de la importancia de la historia en la conformación de la moralidad, con lo cual la dimensión histórica en la obra del autor comienza a ganar presencia.

Después de construir la interpretación kantiana de la vida de Jesús, intentando mostrar que las enseñanzas de éste consistieron en que la razón es el único dejo de divinidad en los hombres y para alcanzar la gracia de Dios, lejos de seguir los preceptos religiosos sin más, el hombre debe llevar siempre a cabo un uso autónomo de su razón, en la segunda etapa de la época de juventud, que va aproximadamente de 1796 a 1799, Hegel realiza una crítica a la filosofía moral kantiana. En la primera parte de la época juvenil de la que hemos hablado, Hegel cree encontrar la presencia del absoluto en el mundo a través del uso práctico y autónomo de la razón. A la positividad del cristianismo y la objetividad de sus leyes intenta hacerle frente mediante la asunción de una religiosidad subjetiva, la cual parte de la estructura autónoma de la voluntad. Con una religión que sea sentida por lo hombres y no sólo reproducida a través del seguimiento puntual de los mandatos divinos, cree Hegel que es posible superar las escisiones abiertas por el cristianismo entre vida y doctrina. Sin embargo, tras realizar la interpretación del fracaso de la empresa de Jesús por oponer el carácter autónomo de la voluntad a las condiciones heterónomas del pueblo hijo de Abraham, Hegel se aleja de aquel punto de vista sobre el absoluto. Ahora tiene una lectura de la moralidad kantiana no sólo como 
incapaz de lograr las reconciliaciones entre vida y doctrina, razón $\mathrm{y}$ fe, $\mathrm{y}$, por tanto, entre el individuo y la realidad, sino como la generadora de las escisiones y fracturas más importantes del sujeto consigo mismo. Guiado por el ideal de la filosofía de la identidad, que intenta captar la existencia real del absoluto en tanto absoluto, Hegel voltea la mirada de la autonomía moral kantiana al ideal de amor-pasión del romanticismo alemán.

Entre 1797 y 1798, fechas en las que se reúne en Frankfurt con Hölderlin, su viejo amigo de juventud, Hegel introduce a Kant a la misma crítica que antes había dirigido a las religiones positivas, entre las que se encuentra el cristianismo y ahora el proyecto kantiano de una religión de la razón. Se trata de la crítica a Kant que será recurrente sobre todo en la Fenomenología del espíritu y Los principios de la filosofía del derecho, cuya primera aparición se encuentra al final de los «Esbozos de amor y religión» que datan de 1797-1798, y tiene seguimiento en «El amor y la propiedad» de 1798-1799.

Hegel acepta que el valor del pensamiento autónomo es mucho mayor al que tiene una voluntad que es puesta en manos de un tercero para que decida sobre ella, tanto en términos de conocimiento como de moral. Pero la supuesta libertad a la que aspira el pensamiento autónomo a lo más que puede llegar es a que el sujeto esté libre de determinaciones externas, mas no ajenas. La reconciliación de las desgarraduras del mundo no puede darse con la simple traslación de los mandatos que deben ser obedecidos de la exterioridad a la interioridad del ámbito propio de la conciencia. El problema no es si la voz a la que escucha y debe obedecer es la de otro hombre o la suya, la de su propia conciencia; el verdadero problema está en la existencia de una voz que ordena. En un mundo en el que la conciencia está reconciliada con la realidad no es posible la obediencia porque no existe el mandato. La voluntad no tiene frente a ella una ley impuesta, sino que obra de manera fluida con el todo de la comunidad y la vida. Para Hegel, el problema de la autonomía kantiana de la voluntad estriba en que si bien el hombre actúa por sí mismo, lo hace no porque esté fusionado con la vida y desde ahí actúe en unidad con la objetividad y organicidad del mundo, de modo que desaparezca la distancia entre el sujeto y el objeto, entre la particularidad del querer y la obligación moral. El 
hombre actúa de acuerdo a las leyes morales que son dictadas por el juez de su conciencia; la inclinación subjetiva, lo que el hombre quiere, son distintos, e incluso contradictorios, con la objetividad de la ley moral. Para Hegel la única diferencia entre la heteronomía y la autonomía de la voluntad se encuentra en que el mandato, la vigilancia y la coerción son ejercidas desde la interioridad de la conciencia y no desde fuera de ella:

[...] era de esperar que Jesús obrara contra la positividad de los mandamientos morales, contra la mera legalidad; que mostrara la universalidad de lo legal y que toda su obligatoriedad proviene de su universalidad, pues si, por una parte, todo Debe, todo mandamiento se anuncia como algo ajeno, por otra, en cuanto concepto (la universalidad), es algo subjetivo. Era de esperar que explicara que, por esto, la legalidad, en cuanto producto de una fuerza humana, de la Facultad de lo universal, de la razón, pierde toda su objetividad, su positividad, su heteronomía, y que el objeto del mandamiento se manifiesta por lo mismo como fundado en la autonomía de la voluntad humana.

Sin embargo, la positividad desaparece sólo parcialmente por intermedio de este proceso... entre los Shamanos de los Tunguses, los prelados europeos que gobiernan en la Iglesia y el Estado y los puritanos, por una parte, y el hombre que obedece al mandamiento de su propio deber, por otra, la diferencia no está en que los primeros estén en la servidumbre y este último sea libre, sino en que los primeros tienen a su Señor fuera de sí, mientras que el segundo lo lleva dentro de sí mismo, siendo al mismo tiempo su propio esclavo7.

Aquí comienza propiamente la ruptura de Hegel, no con sus intereses teóricos de juventud, sino con la manera de esbozar sus ideas. Su separación respecto del trascendentalismo kantiano da pie a los cimientos del realismo especulativo desde el que un poco más tarde construirá el sistema. Con la crítica a Kant, Hegel se aleja de las posturas que conciben teóricamente un modelo de mundo que desde fuera intentan consolidar en la realidad, tal como el mismo Hegel hace en Berna desde finales de 1795 a través de su interpre-

7 G. W. F. Hegel, «El espíritu del cristianismo y su destino», en: Escritos de juventud, p. 308. 
tación de las enseñanzas de Jesús. La mediación entre la autonomía kantiana de la voluntad ya superada y el proyecto sistemático de reconocer la racionalidad del mundo en sus manifestaciones finitas y reales, tanto pasadas como presentes - proyecto que caracteriza a su sistema a partir de 1800-, pero leído siempre desde su vigencia actual, es el concepto de «amor». Como han interpretado Henrich y Pannenberg, con la noción de amor Hegel anticipa el concepto de «espíritu», pieza central de su sistema especulativo ${ }^{8}$.

En tanto que el absoluto es la re-unión de todas las posibles fracturas y diferencias, tanto de la objetividad del mundo como de la subjetividad de la conciencia y entre ellas, y la autonomía kantiana de la voluntad lejos de reconciliar acentúa las diferencias y fragmenta al sujeto internamente al contraponer la inclinación y el deber moral, entonces la forma en que el absoluto se hace presente en el mundo no puede ser mediante la autonomía de la voluntad racional, sino del amor. «Únicamente en el amor, dice Hegel, somos uno con el objeto: aquí el objeto no domina ni está dominado» ${ }^{9}$. En el amor, los hombres se encuentran en la totalidad armónica en la que no caben las separaciones entre lo subjetivo y lo objetivo; no hay desgarradura entre la conciencia y el mundo, entre los deseos e

8 Así entiende Henrich el paso de Hegel, de la época de juventud a los escritos sistemáticos posteriores. El autor menciona: «De esta comprensión hegeliana del 'amor', como concepto fundamental de su reflexión, brotó sin fisuras el Sistema. El tema del 'amor' fue sustituido, debido a razones que podemos señalar, por la más rica estructura de la 'vida' y, posteriormente, por la del 'espíritu', que tiene más implicaciones que la 'vida'», en: Dieter Henrich, «Hegel y Hölderlin», en: Hegel en su contexto, Caracas: Monte Ávila Editores 1990, p. 23. En su historia de la filosofía, también Pannenberg interpreta así el paso entre las distintas etapas de la obra de Hegel. Según él: «La idea de amor como la superación de las separaciones hechas por el entendimiento - una superación que reconoce, en vez de aniquilar $-\mathrm{y}$, por tanto, como representación de la unidad de la vida, fue el germen de la posterior concepción de Hegel del concepto especulativo», en: Wolfhart Pannenberg, Una historia de la filosofía desde la idea de Dios, Salamanca: Sígueme 2002, p. 268. En el presente texto partimos de la tesis de que aquello que Hegel busca tematizar desde la época de juventud y hasta las últimas lecciones dictadas en Berlín, es la presencia del absoluto. Los conceptos desde los cuales se lo tematiza son los de autonomía kantiana de la voluntad y amor en el caso de la época de juventud; en los escritos sistemáticos posteriores, como muy bien lo entiende Henrich, el concepto de amor es llevado al de espíritu. En el sistema, el espíritu se aprehende en las distintas formas que va adquiriendo hasta llegar a ser saber de sí: saber absoluto.

${ }^{9}$ G. W. F Hegel, «Esbozos sobre religión y amor», en: Escritos de juventud, p. 241. 
inclinaciones subjetivas y los principios objetivos del deber moral. En el amor, la unión de las partes y el todo se fusionan a tal grado que pierde sentido no sólo hablar de partes aisladas, sino de la unificación misma. Una vez en el amor, carece de pertinencia pensar en alguna vinculación entre el sujeto y la vida, o el deber y la inclinación, pues éstos no presentan ninguna escisión porque permanecen siendo uno en el ser. Así, dice Hegel, «La vida ha reencontrado en el amor a la vida» ${ }^{10}$. Dentro de una totalidad bella como la que supone el amor, el hombre no actúa por deber, no hay obligación ni obediencia, así como tampoco hay contradicción alguna entre lo que el sujeto quiere hacer y lo que hace efectivamente. Los sujetos actúan como lo hacen con la plena convicción de que lo quieren, y con el sentimiento de pertenecer a un orden que es suyo. Nos parece que se trata de la antesala a la idea de que «el yo es el nosotros y el nosotros el yo» que aparece en el capítulo de la «autoconciencia» de la Fenomenología del espíritu ${ }^{11}$. Hegel define al amor de la siguiente manera:

El amor no es un universal que se oponga a una particularidad; no es una unidad del concepto, sino unión del espíritu, divinidad. Amar a Dios es sentirse, sin barreras, dentro de la totalidad de la vida, en lo infinito. En ese sentimiento de armonía no hay, por supuesto, universalidad alguna, puesto que en la armonía la particularidad no es discordante, sino concordante ${ }^{12}$.

Bajo esta idea del amor, Hegel comprende al absoluto como unidad indiferenciada. En ella, los amantes se encuentran hundidos en la unidad del ser, unidos en la sustancialidad de la vida reproduciéndola a través de la procreación. Hay aquí una ausencia total de conflicto y diferencia. Hacia el final de los «Esbozos para 'el espíritu del judaísmo'», cuya redacción fue concluida en 1798, Hegel entiende que «Nada se opone más a las bellas relaciones, basadas por naturaleza en el amor, que las de esclavo y señor» ${ }^{13}$. La relación

\footnotetext{
${ }^{10}$ Hegel, «El espíritu del cristianismo...», p. 331.

11 G. W. F. Hegel, Fenomenología del espíritu, México, FCE, 2002, p. 113.

12 Hegel, «El espíritu del cristianismo...», p. 338.

13 G. W. F. Hegel, «Esbozos para <El espíritu del judaísmo»», en: Escritos de juventud,
} 
conflictiva entre el señor y el siervo, tema central de la Fenomenología del espíritu, se opone aquí al amor y, por tanto, a la divinidad misma. La idea del amor le permite a Hegel, en este momento, no sólo superar las escisiones abiertas por las religiones positivas primero, y por el kantismo después, sino mostrar la presencia del absoluto mediante la unidad indiferenciada de las individualidades que se encuentran dentro del mundo, la historia y la comunidad. El amor es ese trasfondo gracias al cual los hombres se vinculan de manera armónica entre sí y con la totalidad estando en continuidad con la naturaleza, de tal modo que toda oposición y relación conflictiva desaparece.

De manera poco sistemática en los escritos de la época de Frankfurt, Hegel comparte con Hölderlin la vinculación entre belleza, amor, naturaleza y religión. Cree que sólo ahí donde la naturaleza y la libertad, el objeto y el sujeto, no se encuentran escindidos está realmente la divinidad. En este espacio de no diferenciación no hay lugar para la oposición entre señor y siervo, pues las individualidades no se han enfrentado de manera conflictiva en búsqueda del reconocimiento de su libertad por oposición a la no libertad de la otra conciencia. Dada la unidad y no separación, no hay relación alguna entre dominado y dominante, pues, dice Hegel, «Sólo puede producirse amor hacia aquello que es igual a nosotros, hacia el espejo, hacia el eco de nuestro ser» ${ }^{14}$. Como hemos mencionado, el resultado de la unidad indiferenciada que es el amor, en el cual, para Hegel «lo separado subsiste todavía, pero ya no como separado, sino como unido; y lo viviente siente a lo viviente» ${ }^{15}$, es la generación y reproducción de la vida a partir de la vida misma, es decir, a través de la procreación. La generación de la vida a partir de la vida, que únicamente puede desprenderse de la fusión unificadora en la que se encuentran los cuerpos de los amantes atravesados por el amor, es la más perfecta muestra de la manera en que la divinidad se hace presente en el mundo. Y lo único que puede romper esta unidad perfecta y bella que es el amor, es

p. 236.

14 Hegel, «Esbozos sobre religión...», p. 242.

15 G. W. F. Hegel, «El amor y la propiedad», en: Escritos de juventud, p. 263. 
la muerte. El amor es vida, nacimiento y reproducción de la naturaleza; la muerte es la negatividad de la vida, el rompimiento de la unidad originaria. «Afirmar que los amantes tienen [cada cual] su independencia, dice Hegel, sus principios propios de vida significa afirmar únicamente que pueden morir» ${ }^{16}$. Es clara aquí la oposición de la unidad entre el amor, la vida y la divinidad frente a la ruptura de la unión, la diferencia y la muerte.

La divinidad, pues, a través del amor se manifiesta como afirmación de la vida. Sin embargo, el amor resulta insuficiente para mostrar su existencia. A pesar de que a través de él Hegel puede acercarse a la tan buscada noción de totalidad, mediante la cual en el sistema reconstruirá el camino de la divinidad en el proceso de formación de sí misma como absoluto, este proceso de formación es netamente humano, y las relaciones basadas en el amor se encuentran aún enraizadas en sentimientos naturales. Ya desde los primeros escritos de juventud se puede ver la manera en que se va perfilando en el pensamiento de Hegel la sugerencia más importante con la que abre la Fenomenología del espíritu: «que lo verdadero no se aprehenda y se exprese como sustancia, sino también y en la misma medida como sujeto» ${ }^{17}$.

Como hemos visto, para Hegel, no hay un ámbito realmente práctico en la filosofía kantiana por el alejamiento radical de la realidad al que nos expone la estructura formal de la moralidad. Pero en el amor tampoco hay lugar para una praxis verdaderamente humana, pues el hombre se encuentra hundido en la totalidad de la naturaleza sin siquiera poder diferenciar su individualidad y autoconformarse como persona. Lo que lleva a la persona a superarse en algo más que un simple ser viviente, es el saberse libre. Distintos elementos intervienen en este proceso, como es el caso de hacer uso de la propiedad privada y la formación familiar que lleva al hijo a separarse del ámbito nuclear para entrar al de la sociedad civil, entre otros. Pero hay uno privilegiado a partir del cual se da el ver-

16 Hegel, «El amor y...», p. 263. Una excelente revisión del papel de la muerte en la concepción hegeliana se puede ver en: Alexandre Kojève, La dialéctica de lo real y la idea de la muerte en Hegel, Buenos Aires: La Pléyade 1984.

17 Hegel, Fenomenología del espíritu, p. 15. 
dadero paso de la naturaleza a la humanidad: la lucha a muerte por el reconocimiento. Únicamente a partir de ella es que el hombre puede construir un mundo verdaderamente humano, sólo a través del cual le es posible proyectar - como muy bien lo deja ver Hegel principalmente en la Fenomenología - su finitud hacia la infinitud del absoluto conformándose como un ser libre. Este es el punto decisivo del hegelianismo a partir aproximadamente de 1800: que la divinidad ya no se manifiesta como afirmación de la vida, sino como negación de la vida en favor de la libertad. Es por esto que la versión romántica del amor, así como el movimiento romántico en general, resultan para Hegel insuficientes para mostrar la presencia y desarrollo del absoluto en el mundo, pues se quedan en un estadio inferior del despliegue de la conciencia en el que el hombre no ha podido enajenarse de su situación natural en el mundo para constituirse como humanidad. En el amor, lo divino sólo puede ser aprehendido como unidad sin poder describir aún sus expresiones fenoménicas mediante lo múltiple. Se trata de un absoluto en todo caso estático y carente de vida. Por el contrario, la vida implica movimiento, enajenación, negación, superación y asunción; aspectos éstos que formarán el movimiento dialéctico al que Hegel hace alusión con el término Aufhebung, mediante el cual es posible ir aprehendiendo paulatinamente el proceso de formación de la totalidad. Con la lucha a muerte por el reconocimiento, es posible la materialización de la persona en su estado de independencia, que en el amor era imposible por la unidad indiferenciada que consumía las individualidades de los amantes. Ahora hay posibilidades para el reconocimiento, pues existe una relación de alteridad a partir de la cual las conciencias se escinden como partes constitutivas de una unidad natural, y se reconocen una frente a la otra para regresar a sí y autoreconocerse. Se trata del movimiento que sale de la naturaleza del yo para encontrarse con un otro y regresar a sí a partir de esa alteridad, reconociéndose a sí mismo sólo gracias a la relación intersubjetiva establecida con aquel otro. En el amor-pasión romántico no hay posibilidades para el reconocimiento ni del yo, ni del otro, ni de mí mismo a partir del otro porque no hay lugar para la diferencia. No hay lugar para la intersubjetividad porque realmente no lo hay ni para la subjetividad, pues las individualidades 
se han perdido como tales en la fusión con una totalidad abarcada por la naturaleza.

Vida (y por ende amor) - comenta Eugenio Trías - , es algo que hunde a la autoconciencia en la servidumbre. La libertad se alcanza negando la vida. $\mathrm{O}$, como dirá [Hegel] en la Fenomenología, arriesgándola. Es la muerte, no la vida, la que determina la espiritualización, la que hace posible al sujeto ser libre: el juego con la muerte. Es en el miedo y en la angustia ante la muerte como el esclavo comienza a liberarse de sus cadenas. En el vencimiento del miedo está la fuerza de la que se espera libertad ${ }^{18}$.

Lejos, entonces, de que la presencia del absoluto se muestre en la unidad armónica que representa el amor, en la cual las individualidades se encuentran identificadas una con la otra hasta perder sus identidades separadas, ahora sólo es posible a partir de su contrario: la lucha a muerte. Mientras que en el amor-pasión romántico, no hay posibilidad para la alteridad porque no hay ninguna diferencia que pueda ser reconocida, en el paso a la comprensión del absoluto a través de la lucha a muerte, hay reconocimiento de la diferencia a través de la oposición, pero no hay identidad ni unidad indiferenciada de los amantes. Lo único que se enfatiza es la diferencia entre particularidades que se enfrentan en favor de la elevación de la certeza de la libertad que cada una tiene de sí en verdad.

Es hasta el tercer momento, el del sistema a partir de 1800, que Hegel puede llevar a cabo una aprehensión de la divinidad como absoluto, donde no sólo se halle la parcialidad de la mismidad divina que no se ha escindido de la naturaleza, sino que capte la salida de sí y su reconocimiento a través de la dialéctica con lo otro de sí, con una conciencia que le hace frente. De esta manera, por otro

18 Eugenio TríAs, El lenguaje del perdón. Un ensayo sobre Hegel, Barcelona: Anagrama 1981, 103-104 pp. Al respecto, Lukács menciona: «Hegel ve en ella [en la categoría de amor] la culminación de la vida, la verdadera superación de todo lo muerto y positivo del mundo, pero ve al mismo tiempo que tampoco en ese sentimiento puede basarse una realidad superior que realmente pueda contraponerse a la positividad de la sociedad burguesa», en: LukÁcs, El joven Hegel..., p. 139. 
lado, puede aprehender conceptualmente el movimiento del espíritu en el proceso de formación de sí mismo a través de su constitución como sujeto, como humanidad que se ha podido elevar por encima de las determinaciones naturales, incluso del amor, y se ha desarrollado como libertad en la historia. A partir de 1800, no hay lugar ya para una comprensión «positiva» del absoluto sino sólo negativa. En los primeros fragmentos para La constitución de Alemania, redactados entre 1798 y 1800, el autor menciona:

El sentimiento de contradicción entre la naturaleza y la vida existente es la necesidad de que sea superada esta contradicción. La cual se supera [en el momento] en que la vida existente ha acabado de perder su poder y su dignidad, en el momento en que se ha convertido en algo puramente negativo ${ }^{19}$.

El sistema filosófico que se irá gestando a partir del nacimiento del siglo XIX, del que Hegel habla en su carta a Schelling de noviembre de 1800, parece en sí mismo un movimiento hegeliano, pues en él Hegel no hace otra cosa que negar los dos momentos anteriores bajo los que creía comprender la presencia del absoluto en el mundo - el absoluto como unidad indiferenciada y como reconocimiento de la diferencia - para superarlos asumiéndolos. Aquí es donde desembocan los trabajos hegelianos de juventud fungiendo, a nuestro parecer, como prolegómenos al sistema hegeliano.

\section{RESUMEN}

El autor reconstruye y ordena el camino que Hegel recorrió en su juventud hacia la formación del sistema filosófico que data, aproximadamente, de 1800; nos ofrece las directrices para una comprensión más cabal del sistema hegeliano así como de la figura de su autor; uno de los grandes teólogos de la modernidad.

Palabras clave: Hegel; Escritos de juventud; Fenomenología del espíritu; amor; reconocimiento.

19 G. W. F. Hegel, «La constitución alemana», en: Escritos de juventud, p. 392. 


\section{ABSTRACT}

The author reconstructs and orders the road that Hegel traveled in his youth towards the formation of his philosophical system that dates to approximately 1800, offers guidelines for a better understanding of the Hegelian system as well as the figure of its author, one of the great theologians of modernity.

Key words: Hegel; Early Theological Writings; Phenomenology of Spirit; love; recognition. 
